\title{
Outbreaks of carbapenem-resistant Enterobacteriaceae infections associated with duodenoscopes: What can we do to prevent infections?
}

\author{
William A. Rutala PhD, MPH ${ }^{a, b, *}$, David J. Weber MD, MPH ${ }^{a, b}$ \\ ${ }^{a}$ Hospital Epidemiology, University of North Carolina Health Care, Chapel Hill, NC \\ ${ }^{\mathrm{b}}$ Division of Infectious Diseases, UNC School of Medicine, Chapel Hill, NC
}

Key Words:

Endoscopes

disinfection

sterilization

outbreaks

endoscopic retrograde

cholangiopancreatography

duodenoscopes

\begin{abstract}
Recent outbreaks with carbapenem-resistant Enterobacteriaceae (CRE) in patients who have undergone endoscopic retrograde cholangiopancreatography (ERCP) have raised concerns of whether current endoscope reprocessing guidelines are adequate to ensure a patient-safe endoscope. Unlike previous outbreaks, these CRE outbreaks occurred even though manufacturer's instructions and professional guidelines were followed correctly. This article reviews why outbreaks associated with endoscopes continue to occur; what alternatives exist that might improve the margin of safety associated with duodenoscope reprocessing; and how to prevent future outbreaks associated with ERCP procedures. The advantages and disadvantages for the proposed enhancements for reprocessing duodenoscopes are reviewed as well as future strategies to prevent GI endoscope-related outbreaks.
\end{abstract}

In the last 2 years, multiple reports of outbreaks have led the Food and Drug Administration (FDA), Centers for Disease Control and Prevention $(C D C)$, and national news to raise awareness among the public and health care professionals that the complex design of duodenoscopes (used primarily for endoscopic retrograde cholangiopancreatography [ERCP]) may impede effective reprocessing. ${ }^{1,2}$ Several recent publications have associated multidrug-resistant (MDR) bacterial infections, especially those caused by carbapenem-resistant Enterobacteriaceae (CRE), in patients who have undergone ERCP with reprocessed duodenoscopes. ${ }^{3-5}$ Unlike other endoscope outbreaks, these recent outbreaks occurred even when the manufacturer's instructions and professional guidelines were followed correctly., The purpose of this article, which is adapted from recent publications, ${ }^{6,7}$ is 3 -fold: (1) why do outbreaks associated with endoscopes continue to occur; (2) what alternatives exist today that might improve the safety margin as-

\footnotetext{
* Address correspondence to William A. Rutala, PhD, MPH, Hospital Epidemiology, Occupational Health, and Safety Program, Room 1001 West Wing, UNC Health Care, Chapel Hill, NC 27514.

E-mail address: brutala@unch.unc.edu (W.A. Rutala).

Publication of this article was supported by an educational grant from Clorox Healthcare, Sealed Air, and Tru-D. Content of this article was initiated and written by the authors with no input or financial support to the authors from Clorox Healthcare, Sealed Air, or Tru-D.

Conflicts of Interest: Dr Rutala is a consultant for Clorox and has received honoraria from 3M. Dr Weber is a consultant for Clorox.
}

sociated with duodenoscope reprocessing; and (3) how to prevent future outbreaks associated with ERCP endoscopes and other gastrointestinal (GI) endoscopes. ${ }^{6}$

The key concern raised by these outbreaks is that current reprocessing guidelines are not adequate to ensure a patient-safe GI endoscope (one devoid of potential pathogens) because the margin of safety associated with reprocessing endoscopes is minimal or nonexistent. There are 2 (and possibly 3 ) reasons for this reprocessing failure and why outbreaks continue to occur. First, studies have shown that the internal channel of GI endoscopes, including duodenoscopes, may contain $10^{7-10}$ (7-10 $\left.\log _{10}\right)$ enteric microorganisms. ${ }^{8,9}$ Investigations have demonstrated that the cleaning step in endoscope reprocessing results in a 2-6 $\log _{10}$ reduction of microbes, and the high-level disinfection (HLD) step results in another 4-6 $\log _{10}$ reduction of mycobacteria, for a total 6-12 $\log _{10}$ reduction of microbes. ${ }^{8-10}$ Therefore, the margin of safety associated with cleaning and HLD of GI endoscopes is minimal or nonexistent (level of contamination: $4 \log _{10}$ [maximum contamination, minimal cleaning/HLD] to $-5 \log _{10}$ [minimum contamination, maximum cleaning/HLD]). Therefore, any deviation from proper reprocessing (eg, crevices associated with the elevator channel) could lead to failure to eliminate contamination, with a possibility of subsequent patient-to-patient transmission. This low (or nonexistent) margin of safety associated with endoscope reprocessing compares with the $17 \log _{10}$ margin of safety associated with cleaning and sterilization of surgical instruments (ie, $12 \log _{10}$ reduction via sterilization and at least a net $5 \log _{10}$ reduction based on 
microbial load on surgical instruments [2 $\left.\log _{10}\right]$ and microbial reduction via a washer disinfector [7 $\left.\log _{10}\right]$ ).

Second, GI endoscopes not only have a heavy microbial contamination $\left(10^{7}-10^{10}\right.$ bacteria), but they are complex, with long, narrow channels and right angle turns and have difficult to clean and disinfect components (eg, elevator channel). The elevator channel in duodenoscopes is unique to side-viewing endoscopes. It has a separate channel and provides orientation of catheters, guidewires, and accessories into the endoscopic visual field. ${ }^{6,7}$ This channel is complex in design and has crevices that are difficult to access with a cleaning brush and may impede effective reprocessing. ${ }^{2}$ Based on this and other recent studies, it is likely that MDR pathogens are acting as a marker or indicator organism for ineffective reprocessing of the complex design of duodenoscopes, which is an infectious risk to patients. It is unclear if echoendoscopes that have an elevator channel for the same reasons as ERCP scopes (directing accessories) pose the same disinfection challenges and similar infectious risks because these scopes are used to violate otherwise sterile spaces when used to obtain diagnostic samples and for therapeutic interventions.

The third issue that could impact endoscope reprocessing failure and continued endoscope-related outbreaks is the development of a biofilm. ${ }^{11}$ Biofilms are multilayered bacteria plus exopolysaccharides that cement cells to surfaces. They develop in a wet environment. If reprocessing is performed promptly after use and the endoscope is dry, the opportunity for biofilm formation is minimal. ${ }^{12}$ It is unclear if biofilms contribute to failure of endoscope reprocessing.

Given the heavy microbial contamination $\left(10^{7}-10^{10}\right)$ and endoscope components that are difficult to clean and disinfectant (eg, elevator channel), are current endoscope reprocessing guidelines adequate to ensure a GI endoscope devoid of potential pathogens? To examine this question we briefly review the current knowledge on endoscope reprocessing and then offer recommendations. First, endoscopes are semicritical items that require at least HLD. ${ }^{13,14}$ Because flexible GI endoscopes are currently heat labile, only HLD with FDA-cleared high-level disinfectants or lowtemperature sterilization technologies (LTSTs) are possible. ${ }^{13}$ Unfortunately, at present, no solution exists that has been proven to eliminate the risk of microbial contamination associated with duodenoscopes. For example, there is no LTST that achieves a sterility assurance level (SAL) of $10^{-6}$ for GI endoscopes such as duodenoscopes. Second, there have been more health careassociated outbreaks linked to contaminated endoscopes than to any other reusable medical device. ${ }^{13,15}$ However, until recently, these outbreaks have been traced to deficient practices, such as inadequate cleaning, inappropriate disinfection (eg, failure to perfuse all channels), and damaged endoscopes or flaws in the design of endoscopes (eg, duodenoscope elevator channel) or automated endoscope reprocessors. ${ }^{13,15}$ Reprocessing failures have led to patient notifications and bloodborne pathogen testing in dozens of instances. ${ }^{16}$ Third, evidence-based endoscope reprocessing guidelines have been prepared by professional organizations and the CDC, and past data suggested that rigorous adherence to these guidelines would result in a patient-safe endoscope. ${ }^{13,14}$ Unfortunately, there are also data that demonstrate that all of the steps associated with manual endoscope reprocessing are rarely performed and some essential steps (eg, brushing all endoscope channels and components) are uncommonly performed. ${ }^{17}$ Endoscope reprocessing was improved with the use of automated endoscope reprocessors because most steps were automated. ${ }^{17}$ Fourth, endemic transmission of infections associated with GI endoscopes may go unrecognized because of inadequate surveillance of outpatient procedures, the long lag time between colonization and infection, and a low frequency of infection. Additionally, the risk for some procedures might be lower than others (eg, colonoscopy vs ERCP, where normally sterile areas are contaminated in the latter). In the outbreak reported by Wendorf et al, it was the presence of an unusual pathogen (AmpC-producing Escherichia coli) that resulted in an investigation and recognition that duodenoscopes were the source of the outbreak. ${ }^{3}$

What should we do now? Unfortunately, there is currently no single, simple, and proven technology or prevention strategy that hospitals can use to guarantee patient safety. Of course, we must continue to emphasize the enforcement of evidence-based practices, including equipment maintenance and routine audits, with at least yearly competency testing of reprocessing staff. ${ }^{13,14}$ All reprocessing personnel must be knowledgeable and thoroughly trained on the reprocessing instructions for duodenoscopes. This includes the new recommendations to use a small bristle cleaning brush and for additional flushing and cleaning steps of the elevator channel (http://medical.olympusamerica.com/sites/default/files/pdf/150326 _TJF-Q180V_Customer_letter.pdf). Although these steps were described as validated, no public data are available on the ability of these new cleaning recommendations to yield an ERCP scope devoid of bacteria. However, we must do more or additional outbreaks will likely continue. We must obtain additional information on the frequency and level of microbial contamination of endoscopes that have been cleaned and high-level disinfected with strict adherence to current guidelines. If endoscopes are found to be contaminated with potential pathogens (eg, enteric gram-negative bacilli), the clinical impact of such contamination needs to be quantified. In addition, based on the study by Wendorf et al and others, ${ }^{3-5}$ it would be reasonable to consider periodic microbiologic surveillance of duodenoscopes to assess microbial contamination as a component of a prevention strategy; however, culture results are delayed 2-3 days, and there are many questions related to microbiologic surveillance. ${ }^{1}$ These include the following: what cutoff should be used to define proper disinfection (eg, 0 pathogens or a higher number, such as $<10$ colony forming units [CFU] of enteric pathogens per channel)?; should there be a separate cutoff based on relatively nonvirulent pathogens, such as coagulasenegative Staphylococcus?; what sampling scheme should be used to evaluate GI endoscopes (eg, all scopes or a sample of endoscopes)?; if a hospital cultures 2 endoscopes of 10 and 1 endoscope is positive, do they reprocess all 10 endoscopes because $50 \%$ of the sampled endoscopes are positive?; if a hospital does periodic microbiologic culturing and $20 \%$ of sampled endoscopes are positive, what actions should an endoscopy unit undertake (eg, patient notification with an offer of bloodborne pathogen testing, stool examination for CRE, ethylene oxide [ETO] sterilization of positive endoscopes, or HLD followed by ETO sterilization of all duodenoscopes)?; has the staff been trained on culturing the duodenoscope channels and the elevator channel?; and is the trigger for further action based on the level of contamination or the frequency of contamination (ie, percent of endoscopes contaminated) or infection $?^{6,7}$ In addition, if a hospital decides to culture all endoscopes and quarantine endoscopes for 48-72 hours while awaiting culture results before using the scope, it must be recognized that the sensitivity of culturing the elevator channel of the scope or the scope is unknown (ie, how many microbes must contaminate the endoscope to yield a positive culture?). Until there are evidence-based guidelines, individual hospitals should base their decisions on best available information (eg, clinical risk) and what is feasible for theirhospital.

Real-time, monitoring methods need to be developed and validated to assess the effectiveness of cleaning and HLD and the risk of infection. Adenosine triphosphate (ATP) detection of effluent has been offered as a monitoring tool ${ }^{18,19}$ for assessing cleaning because it detects organic residuals. Although ATP may be used to assess endoscope cleaning, it is neither a good indicator of microbial contamination nor validated as a method to assess the risk for patient-to-patient transmission. A validation study of ATP used to audit cleaning of flexible endoscope channels used a benchmark for 
clean ( $<200$ relative light units), which equated to $<4 \log _{10}$ CFU/ $\mathrm{cm}^{2}$ (or $\left.10^{4} \mathrm{CFU} / \mathrm{cm}^{2}\right)^{18}$ or approximately $10^{6} \mathrm{CFU}$ per endoscope because the surface area of an endoscope channel is $>100 \mathrm{~cm}^{2}$. Therefore, an endoscope assessed as clean by ATP could still have a significant microbial load (eg, $\left.10^{6}\right)$.

In regard to the future, we predict that we will continue to see outbreaks associated with ERCP endoscopes and GI endoscopes if we do only the enhanced strategies previously described. One solution to this infection prevention challenge would be to develop new endoscope reprocessing technologies that reliably result in sterilization of duodenoscopes and other GI endoscopes via an FDAcleared sterilization process that achieves an SAL of $10^{-6}$. This SAL results in a $12 \log _{10}$ reduction of microorganisms compared with the $6 \log _{10}$ reduction for HLD. This $6 \log _{10}$ reduction difference is the margin of safety that will ensure endoscopes are devoid of microbial contamination. This can be done by optimizing current LTST (eg, hydrogen peroxide gas plasma, ETO, vaporized hydrogen peroxide) or evaluating new sterilization technologies (eg, ozone plus

Table 1

Summary of advantages and disadvantages of HLD and sterilization enhancements for reprocessing duodenoscopes

\begin{tabular}{|c|c|c|}
\hline Method & Advantages & Disadvantages \\
\hline Steam, sterilization & $\begin{array}{l}\text { - Rapidly microbicidal } \\
\text { - Least affected by organic or inorganic soils among } \\
\text { sterilization processes listed } \\
\text { - Rapid cycle time }\end{array}$ & $\begin{array}{l}\text { - Deleterious for heat-sensitive instruments } \\
\text { - At present they cannot be used because current GI scopes } \\
\text { are not heat resistant }\end{array}$ \\
\hline $\begin{array}{l}\text { Hydrogen peroxide gas plasma, } \\
\text { sterilization }\end{array}$ & $\begin{array}{l}\text { - Cycle time is } \geq 28 \mathrm{~min} \text {, and no aeration necessary } \\
\text { - Used for heat- and moisture-sensitive items because } \\
\text { process temperature }<50^{\circ} \mathrm{C} \\
\text { - Compatible with most medical devices }\end{array}$ & $\begin{array}{l}\text { - Endoscope or medical device restrictions based on lumen } \\
\text { internal diameter and length } \\
\text { - GI scopes cannot be processed } \\
\text { - No microbicidal efficacy data proving SAL } 10^{-6} \text { achieved } \\
\text { - Studies question microbicidal activity in presence of } \\
\text { organic matter and salt } \\
\text { - May damage endoscope }\end{array}$ \\
\hline $\begin{array}{l}\text { 100\% ETO, sterilization after } \\
\text { HLD, microbiologic } \\
\text { surveillance }\end{array}$ & $\begin{array}{l}\text { - Single-dose cartridge and negative- pressure chamber } \\
\text { minimizes the potential for gas leak and ETO exposure } \\
\text { - Simple to operate and monitor } \\
\text { - Compatible with most medical materials } \\
\text { - Major endoscope manufacturer offers ETO as sterilization } \\
\text { option } \\
\text { - Ideally, ETO should be used after standard HLD } \\
\text { - Some data demonstrate reduced infection risk with HLD } \\
\text { followed by ETO }\end{array}$ & $\begin{array}{l}\text { - Requires aeration time to remove ETO residue } \\
\text { - Only } 20 \% \text { of U.S. hospitals have ETO on-site } \\
\text { - Lengthy cycle and aeration time } \\
\text { - No microbicidal efficacy data proving SAL } 10^{-6} \text { achieved } \\
\text { - Studies question microbicidal activity in presence of } \\
\text { - } \text { organic matter and salt } \\
\text { - May is toxic, a carcinogen, flammable }\end{array}$ \\
\hline $\begin{array}{l}\text { Vaporized hydrogen peroxide, } \\
\text { sterilization }\end{array}$ & $\begin{array}{l}\text { - Fast cycle time (55 min) } \\
\text { - Used for heat- and moisture-sensitive items (metal and } \\
\text { nonmetal devices) }\end{array}$ & $\begin{array}{l}\text { - Endoscope or medical device restrictions based on lumen } \\
\text { internal diameter and length } \\
\text { - GI scopes cannot be processed } \\
\text { - No microbicidal efficacy data proving SAL } 10^{-6} \text { achieved } \\
\text { - No data demonstrating microbicidal activity in presence of } \\
\text { organic matter and salt } \\
\text { - May damage endoscope }\end{array}$ \\
\hline $\begin{array}{l}\text { HLD only (using FDA-cleared } \\
\text { HLD, such as OPA and glut) }\end{array}$ & $\begin{array}{l}\text { - HLD inactivate MDR organisms, including CREs } \\
\text { - Current standard of care } \\
\text { - Wide availability }\end{array}$ & $\begin{array}{l}\text { - Based on recent ERCP outbreaks, infection risk related to } \\
\text { device complexity and microbial load } \\
\text { - No enhancement to reduce infection risk associated with } \\
\text { ERCP scopes } \\
\text { - Some HLD (eg, aldehydes) may cross-link proteins }\end{array}$ \\
\hline $\begin{array}{l}\text { Double HLD (back-to-back); } \\
\text { microbiologic surveillance }\end{array}$ & $\begin{array}{l}\text { - HLD inactivate MDR organisms, including CREs } \\
\text { - Wide availability } \\
\text { - A second HLD cycle may reduce or eliminate microbial } \\
\text { contaminants remaining from first cycle }\end{array}$ & $\begin{array}{l}\text { - Based on recent ERCP outbreaks, infection risk related to } \\
\text { device complexity and microbial load } \\
\text { - Some HLD (eg, aldehydes) may cross-link proteins }\end{array}$ \\
\hline $\begin{array}{l}\text { Liquid chemical sterilant } \\
\text { processing system using } \\
\text { peracetic acid, rinsed with } \\
\text { extensively treated potable } \\
\text { water, microbiologic } \\
\text { surveillance }\end{array}$ & $\begin{array}{l}\text { - HLD and chemical sterilant inactivate MDR organisms, } \\
\text { including CREs } \\
\text { - Offered as liquid chemical sterilant processing option }\end{array}$ & $\begin{array}{l}\text { - Based on recent ERCP outbreaks, infection risk related to } \\
\text { device complexity and microbial load } \\
\text { - Not considered sterile because it is not a terminal } \\
\text { sterilization process and scope is rinsed with extensively } \\
\text { treated water } \\
\text { - Unclear if peracetic acid will penetrate crevices in elevator } \\
\text { channel and inactivate pathogens }\end{array}$ \\
\hline $\begin{array}{l}\text { HLD, microbiologic } \\
\text { surveillance }\end{array}$ & $\begin{array}{l}\text { - HLD inactivate MDR organisms, including CREs } \\
\text { - Microbiologic surveillance offered as supplement by the } \\
\text { CDC }\end{array}$ & $\begin{array}{l}\text { - Based on recent ERCP outbreaks, infection risk related to } \\
\text { device complexity and microbial load } \\
\text { - No data demonstrating reduced infection risk } \\
\text { - Sensitivity of microbiologic surveillance unknown } \\
\text { - } 48-72 \text { hours before culture results known } \\
\text { - No consensus regarding sampling scheme, } 100 \% \text { or } 10 \% \text { of } \\
\text { scopes per week or per month? } \\
\text { - No cutoff to define effective disinfection (0 GNR?) }\end{array}$ \\
\hline HLD, ATP & $\begin{array}{l}\text { - HLD inactivate MDR organisms, including CREs } \\
\text { - Real-time monitoring tool } \\
\text { - Simple to conduct } \\
\text { - Detects organic residue }\end{array}$ & $\begin{array}{l}\text { - Based on recent ERCP outbreaks, infection risk is related to } \\
\text { device complexity and microbial load } \\
\text { - No data demonstrating reduced infection risk } \\
\text { - Does not detect microbial contamination } \\
\text { - ATP not validated as risk factor for patient-to-patient } \\
\text { transmission } \\
\text { - Unknown cutoff level to assure safety }\end{array}$ \\
\hline
\end{tabular}

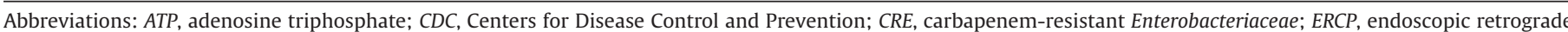

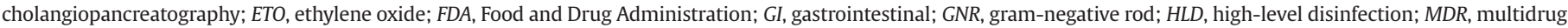
resistant; $O P A$, ortho-phthalaldehyde; $S A L$, sterility assurance level. 
hydrogen peroxide vapor, nitrogen dioxide, ${ }^{20}$ supercritical $\mathrm{CO}_{2}$, peracetic acid vapor, gaseous chlorine dioxide, steam sterilization for heat-resistant GI endoscopes). These new technologies could greatly improve the margin of safety and eliminate patient risk. ETO, which was used by Epstein et al to terminate their outbreak, ${ }^{4}$ may be a short-term solution for some hospitals, but it is not a long-term satisfactory solution because many hospitals no longer have ETO, the sterilization-aeration time is long (eg, 12-15 hours), and the process may eventually damage the endoscopes. Additionally, there are no studies in the peer-reviewed literature that demonstrate that we can depend on ETO (or other LTST) to sterilize a duodenoscope following only the cleaning portion of the endoscope reprocessing instructions (ie, brushing, flushing). In fact, Alfa et al found the sterilization efficacy with $100 \%$ ETO or hydrogen peroxide gas plasma was only $39.7 \%$ and $35 \%$, respectively, when serum and salt load was combined with a lumen carrier as the test challenge. When penicylinders were inoculated with 7 organisms in the presence of salt and serum sterilization efficacy with 100\% ETO and hydrogen peroxide gas plasma was $60.3 \%$ and $37 \%$, respectively. ${ }^{21,22}$ For this reason, if hospitals wish to consider ETO as an enhancement to their current ERCP reprocessing, the scope should be cleaned and highlevel disinfected and dried per reprocessing instruction prior to ETO. Prior cleaning and HLD reduces the microbial load and organic challenge that may interfere with the effectiveness of ETO sterilization. It is unlikely, based on existing data that ETO (or other LTST) will kill high numbers of pathogens in the presence of salt and serum in a lumened device.

Table 1 provides the advantages and disadvantages for various enhancements involving HLD or sterilization. Table 2 offers current enhancements and future sterilization alternatives. Unfortunately, many of these strategies may increase capital equipment and reprocessing costs, cause changes in workflow and processes, and cause a short-term shortage of duodenoscopes used in ERCP procedures. For these reasons and others (including medicallegal), we recommend the infection prevention clinician seek executive-level support for the duodenoscope reprocessing enhancements needed to minimize the infection risk. Other alternative solutions to avoid outbreaks associated with HLD of endoscopes include the following: development of sterile disposable GI endoscopes; improved GI endoscope design to reduce or eliminate the challenges listed in Table 2; or a shift to other sterile diagnostic modalities (eg, capsule endoscopy, blood tests to detect GI cancer).

Improved prevention strategies must be urgently pursued. Despite the very low risk of MDR infection after ERCP, any avoidable infection risk must be eliminated. Manufacturers (for endoscopes, automated endoscope reprocessors, high-level disinfectants, and LTST) and federal authorities (CDC, FDA, National Institutes of Health) must be engaged by providing adequate resources to design and complete the necessary studies for determining the risks posed by current reprocessing of endoscopes and for developing new reprocessing methods and practices. Infection prevention clinicians should be encouraged to report and publish additional outbreaks related to endoscopy, especially if current reprocessing methods were adhered to; therefore, we can determine if the recent reports are the tip-of-the-iceberg or an anomaly. Therefore, infection prevention associated with ERCP and GI scopes is multifaceted, and no single, immediately available strategy will eliminate this problem. However, the immediate risks can be minimized by a multicomponent strategy (eg, compliance with endoscope reprocessing guidelines, HLD followed by ETO, periodic microbiologic sampling). Unfortunately, most hospitals have not implemented the reprocessing enhancements mentioned in Table 1, and approximately one-third reported not using any surveillance method to identify possible infections after duodenoscopy. ${ }^{23}$
Only when we implement new technologies (eg, equipment redesign, single-use sterile endoscopes, sterilization of GI endoscopes with technology that achieves an SAL of $10^{-6}$ ) will we eliminate the risk of infection associated with duodenoscopes and other GI endoscopes.

In May 2015, the FDA convened a panel to discuss recent reports and epidemiologic investigations of the transmission of infections associated with the use of duodenoscopes in ERCP procedures. ${ }^{24} \mathrm{After}$ presentations from industry, professional societies, and invited speakers, the panel made several recommendations to include reclassifying duodenoscopes based on the Spaulding classification from semicritical to critical to support the shift from HLD to sterilization. ${ }^{25}$ Although the panel recommendations are nonbinding, they are used to inform the FDA director, and they will likely result in guidance or regulation for industry. In September 1992, the FDA recommended that reusable dental handpieces and related instruments be heat sterilized between each patient. This followed the first case of dentist-to-patient transmission of HIV; however, this case was not associated with a contaminated dental handpiece. We believe

\section{Table 2}

Challenges in high-level disinfection of GI endoscopes and preventive methods to assure safety

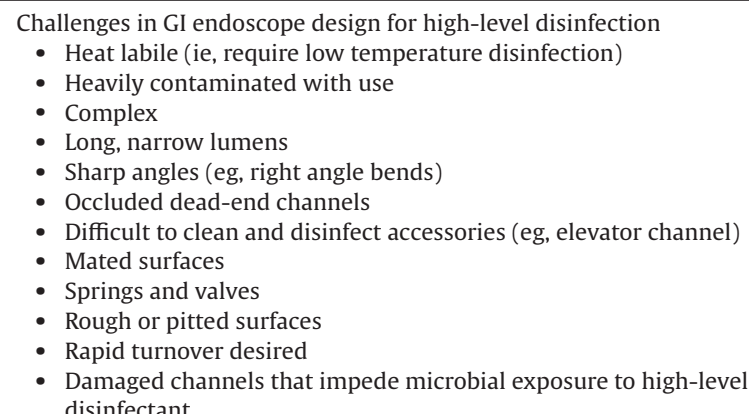
disinfectant

Steps in high-level disinfection (rigorously adhere to current standards for cleaning and disinfection)

- Preclean at point of use

- Clean thoroughly

- High-level disinfection (following manufacturer's recommendations)

- Rinse thoroughly

- Dry completely

- Store properly

- Also assure competency of reprocessing staff (initial and at least annual training assessment)

Possible current enhanced methods for reprocessing duodenoscopes currently available

- Ethylene oxide sterilization after high-level disinfection with periodic microbiologic surveillance

- Double high-level disinfection with periodic microbiologic surveillance

- High-level disinfection with scope quarantine until negative culture

- Liquid chemical sterilant processing system using peracetic acid (rinsed with extensively treated potable water) with periodic microbiologic surveillance

- Other FDA-cleared low-temperature sterilization technology (provided material compatibility and sterilization validation testing performed using the sterilizer and endoscope) after high-level disinfection, with periodic microbiologic surveillance

- High-level disinfection with periodic microbiologic surveillance

Potential future methods to prevent GI endoscope-related outbreaks

- Steam sterilization GI endoscopes

- Optimize existing low temperature sterilization methods or develop new low temperature sterilization methods proving SAL $10^{-6}$ achieved (see text)

- Disposable sterile GI endoscopes

- Improved GI endoscope design (to reduce or eliminate challenges)

- Use of nonendoscope methods to diagnosis or treat disease (eg, capsule endoscopy, blood tests to detect GI cancer, stool, DNA test)

Abbreviations: FDA, Food and Drug Administration; GI, gastrointestinal; SAL, stability assurance level. 
the FDA, in collaboration with industry and infection prevention clinicians, should pursue new infection prevention strategies with urgency and laser-like focus.

It was recommended at the meeting that this could be accomplished by shifting from HLD to sterilization and modifying the Spaulding definition of critical items from "objects which enter sterile tissue or the vascular system or through which blood flows should be sterile" to "objects which directly or secondarily (ie, via a mucous membrane, such as a duodenoscope) enter normally sterile tissue of the vascular system of through which blood flows should be sterile" 7,25 Implementation of these recommendations requires sterilization technology that achieves an SAL of $10^{-6}$ of complex medical instruments, such as duodenoscopes. Ideally, this shift would eventually involve not only endoscopes that secondarily enter normally sterile tissue (eg, duodenoscopes, bronchoscopes) but also other semicritical devices (eg, GI endoscopes).

Until these issues can be resolved, we should continue to provide GI endoscopic (eg, ERCP) procedures, which are an important diagnostic and therapeutic modality. These procedures should be done while strictly adhering to current endoscope reprocessing guidelines $^{3,4}$ with the enhancements offered (Tables 1 and 2) while informing patients of the benefits (ie, potentially life-saving procedure) and risks.

\section{References}

1. Centers for Disease Control and Prevention. Interim duodenoscope surveillance protocol: interim protocol for healthcare facilities regarding surveillance for bacterial contamination of duodenoscopes after reprocessing. Available from: http://www.cdc.gov/hai/organisms/cre/cre-duodenoscope-surveillance-protocol html. Accessed March 27, 2015

2. Food and Drug Administration. Design of endoscopic retrograde cholangiopancreatography (ERCP) duodenoscopes may impede effective cleaning: FDA safety communication. Available from: http://www.fda.gov/MedicalDevices/ Safety/AlertsandNotices/ucm434871.htm. Accessed March 26, 2015.

3. Wendorf KA, Kay M, Baliga C, Weissman SJ, Gluck M, Verma P, et al. Endoscopic retrograde cholangiopancreatography-associated AmpC Escherichia coli outbreak Infect Control Hosp Epidemiol 2015;36:634-42.

4. Epstein L, Hunter JC, Arwady MA, Tsai V, Stein L, Gribogiannis M, et al. New Delhi metallo- $\beta$-lactamase-producing carbapenem-resistant Escherichia coli associated with exposure to duodenoscopes. JAMA 2014;312:1447-55.

5. Carbonne A, Thiolet JM, Fournier S, Fortineau N, Kassis-Chikhani N, Boytchev I, et al. Control of a multi-hospital outbreak of KPC-producing Klebsiella pneumoniae type 2 in France, September to October 2009. Euro Surveill 2010;15:pii: 19734.

6. Rutala WA, Weber DJ. ERCP scopes: what can we do to prevent infections. Infect Control Hosp Epidemiol 2015;36:643-8.

7. Rutala WA, Weber DJ. Gastrointestinal endoscopes: a need to shift from disinfection to sterilization. JAMA 2014;312:1405-6.
8. Roberts CG. Studies on the bioburden on medical devices and the importance of cleaning. In: Rutala WA, editor. Disinfection, sterilization and antisepsis: principles and practices in healthcare facilities. Washington, DC: Association for Professionals in Infection Control and Epidemiology; 2000:63-9.

9. Alfa MJ, Degagne P, Olson N. Worst-case soiling levels for patient-used flexible endoscopes before and after cleaning. Am J Infect Control 1999;27:392-410.

10. Rutala WA, Weber DJ. FDA labeling requirements for disinfection of endoscopes: a counterpoint. Infect Control Hosp Epidemiol 1995;16:231-5.

11. Pajkos A, Vickery K, Cossart Y. Is biofilm accumulation on endoscope tubing a contributor to the failure of cleaning and decontamination. J Hosp Infect 2004;58:224.

12. Roberts CG. The role of biofilm in reprocessing medical devices. Am J Infect Control 2013;41(Suppl):577-80.

13. Rutala WA, Weber DJ, Healthcare Infection Control Practices Advisory Committee. Guideline for disinfection and sterilization in healthcare facilities. Available from: http://www.cdc.gov/hicpac/pdf/guidelines/Disinfection_Nov_2008.pdf. Accessed December 28, 2015.

14. Petersen BT, Chennat J, Cohen J, Cotton PB, Greenwald DA, Kowalski TE, et al. Multisociety guideline on reprocessing flexible GI endoscopes: 2011. Infect Control Hosp Epidemiol 2011;32:527-37.

15. Kovaleva J, Peters FTM, van der Mei HC, Degener JE. Transmission of infection by flexible gastrointestinal endoscopy and bronchoscopy. Clin Microbiol Rev 2013;26:231-53.

16. Rutala WA, Weber DJ. How to assess risk of disease transmission when there is a failure to follow recommended disinfection and sterilization principles. Infect Control Hosp Epidemiol 2007;28:519-24.

17. Ofstead CL, Wetzler HP, Snyder AK, Horton RA. Endoscope reprocessing methods: a prospective study on the impact of human factors and automation. Gastroenterol Nurs 2010;33:304-11.

18. Alfa MJ, Fatima I, Olson N. Validation of adenosine triphosphate to audit manual cleaning of flexible endoscope channels. Am J Infect Control 2013;41:245-8.

19. American Society of Gastrointestinal Endoscopists Technology Committee. Technologies for monitoring the quality of endoscope reprocessing. Gastrointest Endosc 2014;80:369-72.

20. Schneider PM. New technologies and trends in sterilization and disinfection. Am J Infect Control 2013;41:S81-6.

21. Alfa MJ, DeGagne P, Olson N, Puchalski T. Comparison of ion plasma, vaporized hydrogen peroxide, and 100\% ethylene oxide sterilizers to the 12/88 ethylene oxide gas sterilizer. Infect Control Hosp Epidemiol 1996;17:92-100.

22. Alfa MJ, Olson N, DeGagne P, Hizon R. New low temperature sterilization technologies: microbicidal activity and clinical efficacy. In: Rutala WA editor. Disinfection, sterilization and antisepsis in health care. Champlain (NY): APIC, Washington DC and Polyscience Publications; 1998:67-78.

23. Beekmann SE, Palmore TN, Polgreen PM, Bennett JV. Adequacy of duodenoscope reprocessing methods as reported by infectious disease physicians. Infect Control Hosp Epidemiol 2015 Oct 27:1-3. [Epub ahead of print].

24. Department of Health \& Human Services, Food and Drug Administration. Brief summary of the gastroenterology and urology devices panel meeting, May 14-15, 2015. Available from: http://www.fda.gov/downloads/ AdvisoryCommittees/CommitteesMeetingMaterials/MedicalDevices/ MedicalDevicesAdvisoryCommittee/Gastroenterology-UrologyDevicesPanel/ UCM447407.pdf. Accessed December 28, 2015.

25. Rutala WA. ERCP scopes: what can we do to prevent infection. Available from: http://www.fda.gov/downloads/AdvisoryCommittees/CommitteesMeeting Materials/MedicalDevices/MedicalDevicesAdvisoryCommittee/ Gastroenterology-UrologyDevicesPanel/UCM447146.pdf. Accessed December 28, 2015 